\title{
Electrically controllable spin filtering and switching in multiferroic tunneling junctions
}

\author{
Sheng Ju, ${ }^{1,2, *}$ Tian-Yi Cai, ${ }^{2,3}$ Guang-Yu Guo, ${ }^{1, \dagger}$ and Zhen-Ya Li ${ }^{4,2}$ \\ ${ }^{1}$ Department of Physics and Center of Theoretical Sciences, National Taiwan University, Taipei 106, Taiwan \\ ${ }^{2}$ Department of Physics, Suzhou University, Suzhou 215006, China \\ ${ }^{3}$ Institute of Physics, Chinese Academy of Sciences, Beijing 100080, China \\ ${ }^{4}$ CCAST (World Laboratory), P.O. Box 8730, Beijing 100080, China \\ (Received 2 November 2006; revised manuscript received 16 December 2006; published 22 February 2007)
}

\begin{abstract}
We present a theoretical investigation of an electrically controllable spin filter based on multiferroic tunneling junction. This spin filter combines the exchange splitting of ferromagnets and asymmetry in energy potential due to the screening of ferroelectric polarization charges at electrodes. Transfer matrix calculations show an enhanced spin filtering efficiency, depending on the magnitude and orientation of ferroelectric polarization. A transition from a positive tunneling magnetoresistance to a negative one is also found. Furthermore, an electric controllable switching between multiple resistive states via magnetoelectric coupling is also described; this will open a different logic programing in future spintronics.
\end{abstract}

DOI: $10.1103 /$ PhysRevB.75.064419

PACS number(s): 85.75.-d, 73.40.Gk, 77.80.Fm

Spintronics devices require highly spin-polarized electrons. ${ }^{1}$ To achieve this goal, several approaches are currently investigated. The most direct way is to use fully spinpolarized ferromagnets known as half metals, including doped manganites, double perovskite manganites, $\mathrm{CrO}_{2}$, $\mathrm{Fe}_{3} \mathrm{O}_{4}$, and Heussler alloys. ${ }^{2}$ However, the above-mentioned so-called half metals are limited by either the low Curie temperature or the rapid decrease of spin polarization with the increase of temperature, making them extremely difficult to realize in practice. ${ }^{1,2}$ Another way is to use the magneticinsulator-based spin filter. Spontaneously polarized materials through which electrons cross by tunneling may be applied in electronic devices. ${ }^{3-5}$ Multiferroics are unique materials that can display simultaneously electric and magnetic order. $^{6-16}$ The coupling between electric and magnetic orders in multiferroics is a fascinating effect, which is interesting both for its fundamental physics and potential technological applications. In particular, the coexistence of ferroelectric and ferromagnetic orders will provide a unique opportunity for encoding information independently in electric polarization and magnetization to obtain four different logic states. Recently, multiferroic tunneling junctions have been successfully fabricated. ${ }^{17,18}$ These multiferroic junctions are different from a previous proposed case, where a ferroelectric layer is sandwiched between two magnetic semiconducting electrodes. ${ }^{5}$ The first case was demonstrated in a spin-filter device of $\mathrm{Au} / \mathrm{BiMnO}_{3} / \mathrm{La}_{2 / 3} \mathrm{Sr}_{1 / 3} \mathrm{MnO}_{3} .{ }^{17}$ Compared with previous magnetic insulator, $\mathrm{BiMnO}_{3}$ has a much higher $\mathrm{Cu}$ rie temperature as well as a larger exchange splitting. ${ }^{14}$ However, the $50 \%$ tunneling magnetoresistance (TMR) observed at $3 \mathrm{~K}$ is not so significant. ${ }^{17}$ Obviously, ferroelectricity of $\mathrm{BiMnO}_{3}$ should play a crucial role, but the detailed modeling is still absent. On the other hand, multiferroic $\mathrm{BiFeO}_{3}$ has been successfully combined with half-metallic $\mathrm{La}_{2 / 3} \mathrm{Sr}_{1 / 3} \mathrm{MnO}_{3}{ }^{13}$ In contrast to $\mathrm{BiMnO}_{3}, \mathrm{BiFeO}_{3}$ is ferroelectric but weak ferromagnetism. Ferroelectricity alone does not provide spin-polarized electrons. ${ }^{4}$ Nevertheless, it would be interesting and important to know whether the weak ferromagnetism of $\mathrm{BiFeO}_{3}$ will be compensated by its high electric polarization in $\mathrm{BiFeO}_{3}$-based spin filter. Ferromagnetic-ferroelectric $\mathrm{La}_{0.1} \mathrm{Bi}_{0.9} \mathrm{MnO}_{3}$ seems to be a good candidate. However, the strong bias dependence of TMR and the large difference in the resistance between two coercivities (multiple resistive states) in the $\mathrm{La}_{0.1} \mathrm{Bi}_{0.9} \mathrm{MnO}_{3}$-based tunneling junctions make the situation more complicated. ${ }^{18}$ Theoretical understanding of the electron transport through these multiferroic tunneling junctions is of the same importance as previous theoretical approaches for giant magnetoresistance in metallic multilayers and TMR in spin valve. Nonetheless, such a theory is still lacking.

In this paper, we present a theoretical investigation of electron transport through multiferroic tunneling junctions. An ultrathin multiferroic barrier, separating two different metal electrodes [a nonmagnetic metal (NM) and a ferromagnetic conductor (FM)], serves as the spin polarizer. The tunneling conductance is calculated with the standard transfermatrix method. ${ }^{19,20}$ The combination of the screening of polarization charges in metallic electrodes and exchange splitting of barrier is found to lead to an enhanced spinfiltering effect, even in weak ferromagnetic systems. A transition from positive TMR to negative one is also observed. In addition to these fascinating physics resulting from these two independent ferroic orders, an electrically controllable switching between multiple resistive states can be realized, when magnetoelectric coupling is considered. This will open a different logic programing in future spintronics.

The spin-filter tunneling junction is illustrated in Fig. 1. The special feature that differentiates this device from conventional spin filters is the unique potential profile. This electrostatic potential profile is due to the screening of electric polarization charges at electrodes, and its shape depends on the orientation of P. ${ }^{4}$ As shown in Fig. 1, a NM electrode is placed in the left half-space $x<0$, a multiferroic barrier of thickness $d$, and a semifinite FM electrode placed in the right half-space $x>d . m_{L}, m_{B}$, and $m_{R}$ are the effective masses in three regions. $\mu_{L}$ and $\mu_{R}$ are the Fermi energies of the left and right electrodes, respectively. $\Delta_{R}$ and $\Delta_{B}$ represent the exchange splitting of the spin-up and spin-down bands in FM electrode and the multiferroic barrier, respectively. $\varphi_{L}$ and $\varphi_{R}$ are, respectively, the electrostatic potentials at two interfaces relative to the Fermi level $\mu$ of the system. Due to the intrinsic electric polarization of the multiferroic barrier, the 


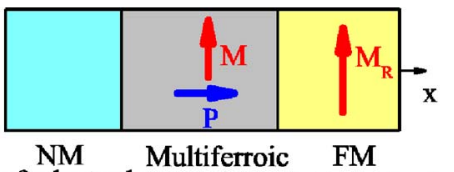

(a) NM Multiferroic FM Left electrode Barrier Right electrode
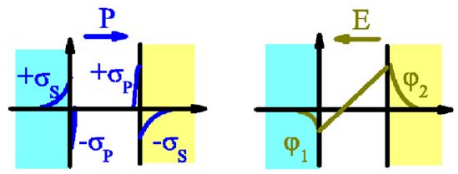

Charge vs. distance Potential vs. distance
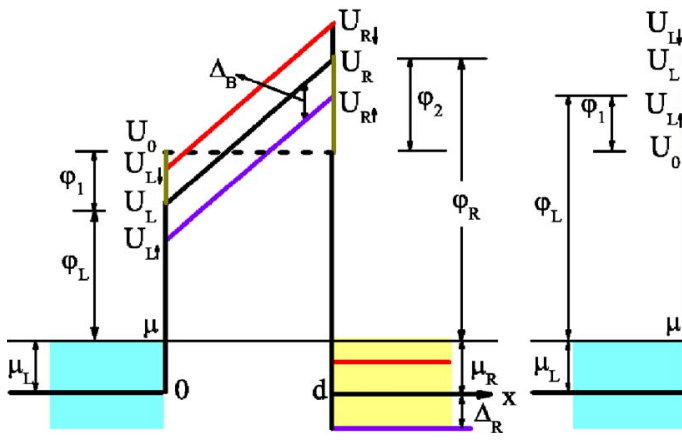

(b) NM Multiferroic FM

Left electrode Barrier Right electrode
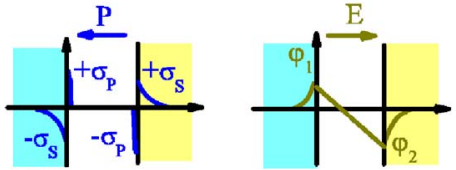

Charge vs. distance Potential vs. distance

built-in bias voltage is $\delta \varphi=\varphi_{L}-\varphi_{R}$. The multiferroic barrier is assumed to be uniformly electrically polarized in the direction perpendicular to the plane. ${ }^{4}$ The electric polarization $\mathbf{P}$ induces surface charge densities, $\pm \sigma_{P}= \pm|\mathbf{P}|$, on the two surfaces of the barrier. These polarization charges, $\pm \sigma_{P}$, are screened by the screening charge per unit area, $\mp \sigma_{S}$, which is induced in the two metallic electrodes. The barrier is assumed to be perfectly insulating and thus all the screening charges reside in the electrodes. According to the ThomasFermi model, the screening potential $\varphi(x)$ is $\sigma_{S} \delta_{L} e^{-|x| / \delta_{L} / \varepsilon_{L}}$ when $x \leqslant 0$ and is $-\sigma_{S} \delta_{R} e^{-|x-d| / \delta_{R} / \varepsilon_{R}}$ when $x \geqslant d .{ }^{4}$ Here, $\delta_{L}$ and $\delta_{R}$ are the Thomas-Fermi screening lengths in the NM and FM electrodes, respectively. $\varepsilon_{L}$ and $\varepsilon_{R}$ are the dielectric permittivities of the NM and FM electrodes. The screening charge $\sigma_{S}$ can be found from the continuity of the electrostatic potential: $\sigma_{S}=\left(d P / \varepsilon_{B}\right) /\left(\delta_{L} / \varepsilon_{L}+\delta_{R} / \varepsilon_{R}+d / \varepsilon_{B}\right)$ and $\varepsilon_{B}$ is the dielectric permittivity of the tunneling barrier.

Accordingly, the overall potential profile is asymmetric, as shown in Fig. 1, because it is the sum of the electrostatic potential $\varphi(z)$, the electronic potential in the electrodes, and the rectangular potential profile $U_{0}$. Under the applied bias voltage $V$, the difference of the interfacial barrier heights is $\delta U=U_{L}-U_{R}=\delta \varphi+e V$, where $U_{L}=\mu+\varphi_{L}$ and $U_{R}=\mu+\varphi_{R}-e V$. Choosing the energy zero of the system so that $\mu=\mu_{L}$, the model Hamiltonian is given by $\hat{H}_{\sigma}=-\left(\hbar^{2} / 2 m_{v}\right) \nabla^{2}+U(x)-\sigma_{v} \Delta_{v}$, with the $x$-dependent potential $U(x)=0$ when $x<0, U(x)=U_{L}-(\delta U / d) x$ when $0 \leqslant x \leqslant d$, and $U(x)=\delta \mu-e V$ when $x>d$, and the spin indices $\sigma_{v}=\theta_{v} \sigma$, where $\sigma$ is the conserved spin orientation in three regions and $v$ indicates $L, B$, or $R . \sigma=+1(\uparrow)$ or $-1(\downarrow)$ means up spin or down spin with respect to $z$. $\theta_{v}=+1(\Uparrow)$ or $-1(\Downarrow)$ denotes the magnetization orientation in the region $v$, parallel or antiparallel to the positive $z$ direction. Then, $\sigma_{v}=+1(\uparrow)$ or $-1(\downarrow)$ is the relative spin orientation, parallel or antiparallel to the given magnetization in the $v$ region. Without loss of generality, we fix $\theta_{B}=+1(\Uparrow)$ and let $\theta_{R}$ vary.
FIG. 1. (Color online) Schematic illustration of our multiferroic spin-filter tunneling junction, the charge distribution and corresponding electrostatic potential (Ref. 4), and the overall potential profile (from top to bottom). (a) The electric polarization $\mathbf{P}$ points to the right (positive). (b) $\mathbf{P}$ points to the left (negative). Here, it is assumed that two electrodes have different screening lengths and $\delta_{1}<\delta_{2}$.
If the eigenenergy $\mathcal{E}$ and the transverse momentum $\hbar q$ are conserved in this structure, the longitudinal energies in three regions can be expressed by $E_{v}=\mathcal{E}-\hbar^{2} q^{2} / 2 m_{v}$. Since $U_{L \sigma_{B}}$ and $U_{R \sigma_{B}}$ are normally larger than $E_{B}$, the asymptotic expansion of Airy's functions gives a good approximation for the transmission coefficient of each spin channel $\sigma$ under a certain magnetization $\theta_{R}=\Uparrow$ or $\Downarrow, 20$

$$
T_{\sigma}^{\Uparrow \theta_{R}}(\mathcal{E}, q)=\frac{16 k_{L} k_{R \sigma_{R}} \kappa_{L \sigma_{B}} \kappa_{R \sigma_{B}} e^{-2 \xi_{\sigma_{B}} d}}{\left(k_{L}^{2}+\kappa_{L \sigma_{B}}^{2}\right)\left(k_{R \sigma_{R}}^{2}+\kappa_{R \sigma_{B}}^{2}\right)},
$$

where the reduced wave vectors are ${ }^{20} k_{L}=\lambda_{L}\left(E_{L}\right)^{1 / 2}, k_{R \sigma_{R}}$ $=\lambda_{R}\left(E_{R}-\delta \mu+e V+\sigma_{R} \Delta_{R}\right)^{1 / 2}, \quad \kappa_{L \sigma_{B}}=\lambda_{B}\left(U_{L \sigma_{B}}-E_{B}\right)^{1 / 2}, \quad$ and $\kappa_{R \sigma_{B}}=\lambda_{B}\left(U_{R \sigma_{B}}-E_{B}\right)^{1 / 2}$, and the decaying WKB wave vector is

$$
\xi_{\sigma_{B}}=\frac{2}{3} \frac{\lambda_{B}}{|\delta U|}\left|\left(U_{L \sigma_{B}}-E_{B}\right)^{3 / 2}-\left(U_{R \sigma_{B}}-E_{B}\right)^{3 / 2}\right| .
$$

Here, $\lambda_{v}=\sqrt{2 m_{e}^{2} / m_{v} \hbar^{2}}(v=L, B$, or $R)$ with $m_{e}$ the freeelectron mass.

When the applied bias voltage $V$ is small and the barrier width $d$ is large, the transmission at the Fermi level $\mu$ with $q=0$ contributes predominantly. Following the conventional treatment, ${ }^{15}$ we obtain the zero-temperature conductance as

$$
G_{\sigma}^{\Uparrow \theta_{R}}=\frac{J_{\sigma}^{\theta_{R}}}{V}=\frac{e^{2}}{8 \pi^{2} \hbar} \frac{\xi_{\sigma_{B}}}{d} T_{\sigma}^{\Uparrow \theta_{R}}(\mu, 0) .
$$

For a certain magnetization configuration, the total conductance is given by the sum of two channels (up spin and down spin): $G^{\Uparrow \theta_{R}}=G_{\uparrow}^{\Uparrow \theta_{R}}+G_{\downarrow}^{\Uparrow \theta_{R}}$. The TMR ratio is defined as $\mathrm{TMR}=1-G^{\Uparrow \downarrow} / G^{\Uparrow \Uparrow}$. To show the spin-filtering effect, we introduce two sub-TMR ratio as $\mathrm{TMR}_{\sigma}=1-G_{\sigma}^{\Uparrow \Downarrow} / G_{\sigma}^{\Uparrow \Uparrow}$, corresponding to the higher barrier $(\sigma=\downarrow)$ and lower barrier $(\sigma=\uparrow)$, respectively. Thus, we can define a ratio $\alpha=G_{\uparrow}^{\Uparrow \Uparrow} /\left(G_{\uparrow}^{\Uparrow \Uparrow}+G_{\downarrow}^{\Uparrow \Uparrow}\right)$ to represent the ratio between conduc- 
tances through the higher and lower barriers. The TMR can be reformulated as $\mathrm{TMR}=\alpha \times \mathrm{TMR}_{\uparrow}+(1-\alpha) \times \mathrm{TMR}_{\downarrow}$. Clearly, when the spin-filtering effect is very strong, $\alpha \rightarrow 1$ $(0)$, then $\mathrm{TMR} \approx \mathrm{TMR}_{\uparrow}\left(\mathrm{TMR}_{\downarrow}\right)$, which means that tunneling electrons are fully spin polarized and TMR is dominated by one of the spin channels. The inverse of spin-filtering ratio can be extended to

$$
\frac{1}{\alpha}=1+\frac{\xi_{\downarrow}}{\xi_{\uparrow}} \frac{k_{R \downarrow} \kappa_{L \downarrow} \kappa_{R \downarrow}}{k_{R \uparrow} \kappa_{L \uparrow} \kappa_{R \uparrow}} \frac{\left(\kappa_{L \uparrow}^{2}+k_{L}^{2}\right)\left(\kappa_{R \uparrow}^{2}+k_{R \uparrow}^{2}\right)}{\left(\kappa_{L \downarrow}^{2}+k_{L}^{2}\right)\left(\kappa_{R \downarrow}^{2}+k_{R \downarrow}^{2}\right)} e^{-2\left(\xi_{\downarrow}-\xi_{\uparrow}\right) d},
$$

and the sub-TMRs can be written as

$$
\mathrm{TMR}_{\sigma}=\frac{k_{R \sigma}-k_{R \bar{\sigma}}}{k_{R \sigma}} \frac{\kappa_{R \sigma}^{2}-k_{R \sigma} k_{R \bar{\sigma}}}{\kappa_{R \sigma}^{2}+k_{R \bar{\sigma}}^{2}},
$$

where $\bar{\sigma}=-\sigma$. If spin-filtering effect is very strong, TMR $\approx \mathrm{TMR}_{\uparrow}=P_{R \uparrow} P_{B \uparrow}$, where $P_{R \uparrow}=\left(k_{R \uparrow}-k_{R \downarrow}\right) / k_{R \uparrow}$ and $P_{B \uparrow}$ $=\left(\kappa_{R \uparrow}^{2}-k_{R \uparrow} k_{R \downarrow}\right) /\left(\kappa_{R \uparrow}^{2}+k_{R \downarrow}^{2}\right)$ are effective spin polarization, respectively. Because of the positive $P_{R \uparrow}$, the sign of TMR is dominated by the term in $P_{B \uparrow}$,

$$
\kappa_{R \uparrow}^{2}-k_{R \uparrow} k_{R \downarrow} \propto \lambda_{B}\left(\varphi_{R \uparrow}-e V\right)-\lambda_{R} \sqrt{\left(\mu_{R}+e V\right)^{2}-\Delta_{R}^{2}},
$$

which is related with the barrier height $\varphi_{R \uparrow}$ induced by electric polarization.

To have some quantitative results of the above arguments, we have calculated the spin filtering efficiency as a function of the exchange splitting, dielectric constant, and thickness of barrier in Figs. 2(a)-2(c), respectively. Shown in the insets are corresponding TMR ratios. Because of the limited available measurements of parameters of the multiferroic thin films, the parameters used in this work to describe ferroelectric properties of the barrier, the barrier height, the screening lengths of metallic electrodes, and magnetic properties of barrier and the right magnetic electrode are chosen to be within the typical values of ferroelectric and magnetic materials. In particular, $U_{0}$ is set to $0.5 \mathrm{eV}$, which is the typical value of ferroelectric insulator. ${ }^{21}$ The dielectric constant of the barrier is around 2000, which represents the value of perovskite ferroelectrics. ${ }^{22} \delta_{L}$ is $0.07 \mathrm{~nm}$, the typical value of a good metal. ${ }^{4}$ For magnetic metal and resistive manganites, the screening length $\delta_{R}$ is larger, and varies up to $1 \mathrm{~nm} .{ }^{23}$ For the exchange splitting in multiferroic barrier and right magnetic electrode, we use the values from Ref. 5. From Fig. 2, one can see immediately that a significant enhancement of spin-filtering efficiency can be achieved even in the case of weak ferromagnetism, provided that $P$ is positive. Three cases are plotted, from top to bottom, $P=100,0$, $-100 \mu \mathrm{C} / \mathrm{cm}^{2}$, respectively. The effects of electric polarization become more important when the barrier is thicker and its dielectric constant is small. The electric polarization dependence of $\alpha$ is given in Fig. 2(d). On the other hand, the sign of TMR, as determined by Eq. (6), has been displayed explicitly in Figs. 3(a) and 3(b), where one can find that either a larger magnitude of electric polarization or a stronger contrast between the screening lengths of two electrodes will lead to a significant decrease of TMR, even to a negative one. From these results, one can conclude that the experimentally observed 50\% TMR in
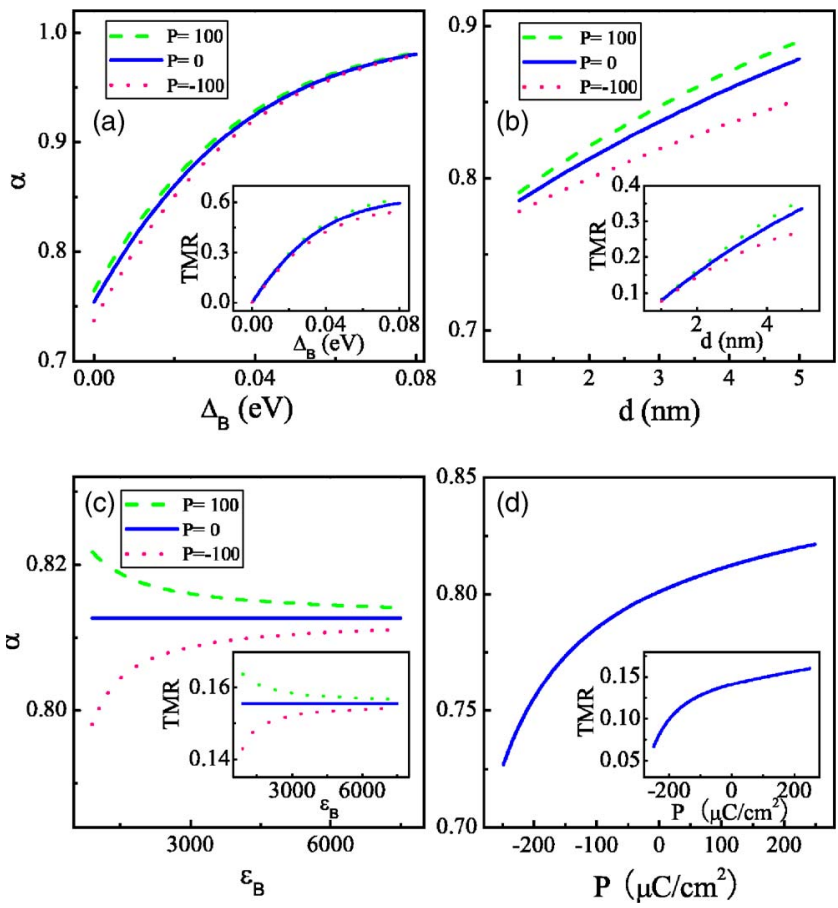

FIG. 2. (Color online) (a) The exchange splitting dependence of spin-filtering efficiency with $\varepsilon_{B}=1000$ and $d=2 \mathrm{~nm}$. (b) Dielectric constant dependence of spin-filtering efficiency with $\Delta_{B}=0.01 \mathrm{eV}$ and $d=2 \mathrm{~nm}$. (c) The thickness dependence of spin-filtering efficiency of the barrier with $\varepsilon_{B}=1000$ and $\Delta_{B}=0.01 \mathrm{eV}$. (d) The $\mathbf{P}$ dependence of spin-filtering efficiency with $\varepsilon_{B}=1000, \Delta_{B}=0.01 \mathrm{eV}$, and $d=2 \mathrm{~nm}$. In the calculation, we set $\delta_{L}=0.07 \mathrm{~nm}, \quad \delta_{R}=0.08 \mathrm{~nm}, \quad \Delta_{R}=0.09 \mathrm{eV}, \quad E_{F}=0.1 \mathrm{eV}, \quad m_{L}=m_{R}$ $=0.9 m_{e}, m_{B}=1.1 m_{e}$, and $U_{0}=0.5 \mathrm{eV}$.

$\mathrm{Au} / \mathrm{BiMnO}_{3} / \mathrm{La}_{2 / 3} \mathrm{Sr}_{1 / 3} \mathrm{MnO}_{3}$ does not necessarily indicate a suppressed exchange splitting in $\mathrm{BiMnO}_{3}$ or a reduced spinfiltering efficiency. ${ }^{17}$ For the ferroelectric degree of freedom in multiferroic barrier, spin-polarization dependence of TMR is more complex than Julliere's model. ${ }^{24}$ As the screening length is considerably large in spin-polarized materials, especially for half metals, ${ }^{23}$ one can conclude that it is the combination of the "wrong" orientation of electric polarization and large contrast between the screening lengths of $\mathrm{Au}$
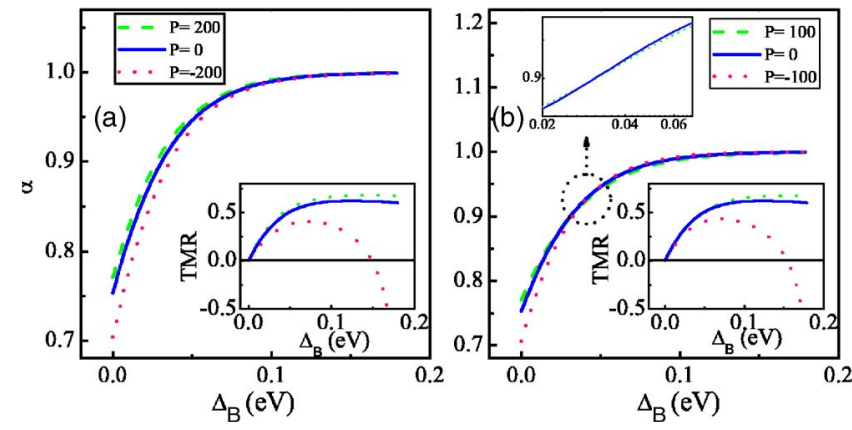

FIG. 3. (Color online) (a) The exchange splitting dependence of spin filtering efficiency for different P. $\delta_{L}=0.07 \mathrm{~nm}$ and $\delta_{R}$ $=0.08 \mathrm{~nm}$. The inset shows the corresponding TMR. (b) The same with (a) but with a stronger contrast between $\delta: \delta_{L}=0.07 \mathrm{~nm}$ and $\delta_{R}=1 \mathrm{~nm}$. 


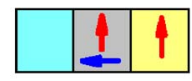

(a)

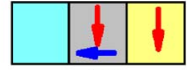

(a')

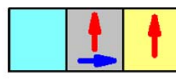

(b)

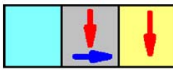

(b')

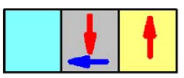

(c)

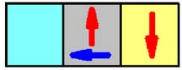

(c')

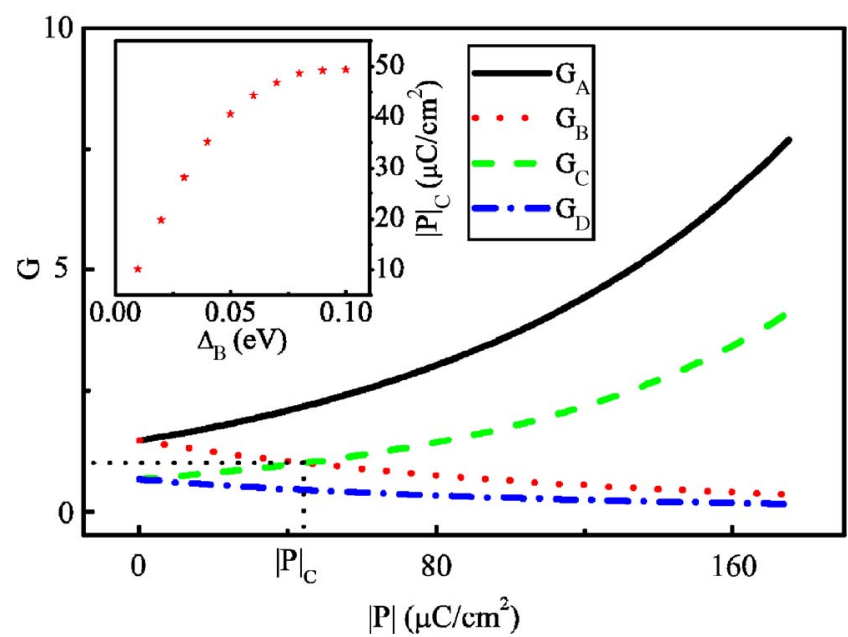

FIG. 4. (Color online) (Upper panel) Schematic illustration of multiple resistive states, depending on the orientation of $\mathbf{P}, \mathbf{M}$ and $\mathbf{M}_{\mathbf{R}}$. (Lower panel) The electric polarization dependence of normalized conductance for the four resistive states. $\delta_{L}=0.07 \mathrm{~nm}, \delta_{R}$ $=1 \mathrm{~nm}, \varepsilon_{B}=2000$, and $\Delta_{B}=0.06 \mathrm{eV}$. The inset shows the $\Delta_{B}$ dependence of $P_{c}$.

and $\mathrm{La}_{2 / 3} \mathrm{Sr}_{1 / 3} \mathrm{MnO}_{3}$ that leads to the low TMR in $\mathrm{Au} / \mathrm{BiMnO}_{3} / \mathrm{La}_{2 / 3} \mathrm{Sr}_{1 / 3} \mathrm{MnO}_{3} .{ }^{17}$ However, using Julliere's model, ${ }^{24}$ a suppressed spin polarization (22\%) (Ref. 17) is deduced from such a 50\% TMR. Such an electric polarization dependence of TMR was observed in a later experiment on $\mathrm{Au} / \mathrm{La}_{0.1} \mathrm{Bi}_{0.9} \mathrm{MnO}_{3} / \mathrm{SrTiO}_{3} / \mathrm{La}_{2 / 3} \mathrm{Sr}_{1 / 3} \mathrm{MnO}_{3}$, where an almost $90 \%$ TMR was observed and the value decreases rapidly with the increase of bias voltage. ${ }^{18}$

With the above consideration, we now discuss the functionality of this FM junction. Previously proposed magnetoelectric spintronics devices are based on the magnetic moment induced by an electric field as $M \propto E{ }^{25}$ The intrinsic electric and magnetic polarizations in multiferroics as described above should lead to somewhat another device concepts. As displayed in Fig. 4 (upper panel), there are overall eight resistive states with four independent pairs $\left(\mathrm{A}, \mathrm{A}^{\prime} ; \mathrm{B}\right.$, $\mathrm{B}^{\prime} ; \mathrm{C}, \mathrm{C}^{\prime}$; and $\mathrm{D}, \mathrm{D}^{\prime}$ ), depending on the relative orientation of neighboring magnetizations and the sign of $\mathbf{P}$. Because of the magnetoelectric coupling in the multiferroics, the $\mathbf{P}$ and the $\mathbf{M}$ can be reversed by an electric field separately or simultaneously. Thus, electric-field controlled functionality can be realized, including normal electroresistance (the transition from $\mathrm{A}$ to $\mathrm{B}$ or the transition from $\mathrm{C}$ to $\mathrm{D}$ ) and more significant change in resistance, i.e., electromagnetoresistance (the transition from A to D). The difference between these states is complex but important for practical application. In the lower panel of Fig. 4, we show the resistance (normalized to its value at $P_{c}$ ) as a function of electric polarization, and the inset displays the exchange splitting dependence of $P_{c}$, where states $\mathrm{B}$ and $\mathrm{C}$ cross. Compared with conventional tunneling magnetoresistive elements which have been applied in magnetic random access memory, and also with recently proposed ferroelectric junctions, the present multiferroic structure possesses both electric controllable switching and large contrast between resistive states.

It should be noted that in this work, we consider only the general aspects of multiferroic junctions (the exchange splitting and ferroelectric polarization of multiferroic barrier) and the results are qualitatively valid. The band structure of specific barrier and electrode, the charge distribution and chemical bonding at certain interfaces, and the magnetoelectric coupling in specific multiferroics can be considered explicitly in future first-principles calculations. In particular, the spiral magnetic structure in $\mathrm{BiFeO}_{3}$, the orbital ordering ferromagnetism in $\mathrm{BiMnO}_{3}$, and the noncollinear magnetism in $\mathrm{TbMnO}_{3}$ may play an important role on the electron tunneling across their junctions, ${ }^{9}$ the detailed calculation of which is, nonetheless, beyond the scope of this paper. In the meantime, in addition to the effect of the screening potential on the electron tunneling, other mechanisms, e.g., the effects of strain and graded polarization at the surface, may be effective for some particular multiferroic barrier. In ferroelectric tunneling junctions, it is found that the effect of strain will lead to the variation of the barrier width, and consequently, change the tunneling conductance. ${ }^{26}$ The graded polarization at the surface is found to enhance the asymmetry of the screening potential, ${ }^{27}$ and consequently, an enhancement of the tunneling electroresistance may be expected. All these mechanisms would perhaps give a further enhancement of the electrically controlled functionalities in multiferroicsbased devices.

To summarize, we have investigated the electron transport through a spin-filter device that uses multiferroics as the tunneling barrier. The interplay between ferroelectric and ferromagnetic degrees of freedom gives rise to an enhanced spinfiltering efficiency, even for the weak ferromagnetic systems. In the mean time, an electric-field controllable switching between multiple resistive states is realized, which should play an important role in the future spintronics.

The authors thank Qian Niu and Junren Shi for their useful suggestions and critical reading of the manuscript. They gratefully acknowledge support from the National Natural Science Foundation of China under Grants Nos. 10474069 and 10504023, National Science Council of Taiwan, and National Center for Theoretical Sciences of Taiwan. 
*Electronic address: jusheng@ @uda.edu.cn

'Electronic address: gyguo@phys.ntu.edu.tw

${ }^{1}$ I. Zutic, J. Fabian, and S. Das Sarma, Rev. Mod. Phys. 76, 323 (2004).

${ }^{2}$ M. Ziese, Rep. Prog. Phys. 65, 143 (2002).

${ }^{3}$ E. Y. Tsymbal and H. Kohlstedt, Science 313, 181 (2006).

${ }^{4}$ M. Y. Zhuravlev, R. F. Sabirianov, S. S. Jaswal, and E. Y. Tsymbal, Phys. Rev. Lett. 94, 246802 (2005).

${ }^{5}$ M. Ye. Zhuravlev, S. S. Jaswal, E. Y. Tsymbal, and R. F. Sabirianov, Appl. Phys. Lett. 87, 222114 (2005).

${ }^{6}$ M. Fiebig, J. Phys. D 38, R123 (2005).

${ }^{7}$ N. A. Spaldin and M. Fiebig, Science 309, 391 (2005).

${ }^{8}$ Y. Tokura, Science 312, 1481 (2006).

${ }^{9}$ W. Eerenstein, N. D. Mathur, and J. F. Scott, Nature (London) 442, 759 (2006).

${ }^{10}$ J. Wang, J. B. Neaton, H. Zheng, V. Nagarajan, S. B. Ogale, B. Liu, D. Viehland, V. Vaithyanathan, D. G. Schlom, U. V. Waghmare, N. A. Spaldin, K. M. Rabe, M. Wuttig, and R. Ramesh, Science 299, 1719 (2003).

${ }^{11}$ W. Eerenstein, F. D. Morrison, J. Dho, M. G. Blamire, J. F. Scott, and N. D. Mathur, Science 307, 1203a (2005).

${ }^{12}$ J. Wang, A. Scholl, H. Zheng, S. B. Ogale, D. Viehland, D. G. Schlom, N. A. Spaldin, K. M. Rabe, M. Wuttig, L. Mohaddes, J. Neaton, U. Waghmare, T. Zhao, and R. Ramesh, Science 307, 1203b (2005).

${ }^{13}$ H. Bea, M. Bibes, M. Sirena, G. Herranz, K. Bouzehouane, E. Jacquet, S. Fusil, P. Paruch, and M. Dawber, Appl. Phys. Lett. 88, 062502 (2006).
${ }^{14}$ T. Kimura, S. Kawamoto, I. Yamada, M. Azuma, M. Takano, and Y. Tokura, Phys. Rev. B 67, 180401(R) (2003).

${ }^{15}$ W. Eerenstein, F. D. Morrison, J. F. Scott, and N. D. Mathur, Appl. Phys. Lett. 87, 101906 (2005).

${ }^{16}$ M. Gajek, M. Bibes, A. Barthelemy, M. Varela, and J. Fontcuberta, J. Appl. Phys. 97, 103909 (2005).

${ }^{17}$ M. Gajek, M. Bibes, A. Barthelemy, K. Bouzehouane, S. Fusil, M. Varela, J. Fontcuberta, and A. Fert, Phys. Rev. B 72, 020406(R) (2005).

${ }^{18}$ M. Gajek, M. Bibes, M. Varela, J. Fontcuberta, G. Herranz, S. Fusil, K. Bouzehouane, A. Barthelemy, and A. Fert, J. Appl. Phys. 99, 08E504 (2006).

${ }^{19}$ T. Y. Cai, Sheng Ju, and Zhen-Ya Li, Phys. Rev. B 71, 224417 (2005).

${ }^{20}$ D. F. Jin, Y. Ren, Z. Z. Li, M. W. Xiao, G. J. Jin, and A. Hu, Phys. Rev. B 73, 012414 (2006).

${ }^{21}$ J. Rodriguez Contreras, H. Kohlstedt, U. Poppe, R. Waser, C. Buchal, and N. A. Pertsev, Appl. Phys. Lett. 83, 4595 (2003).

${ }^{22}$ G. A. Samara, in Solid State Physics, edited by H. Ehrenreich and F. Spaepen (Academic, San Diego, 2001), Vol. 56, p. 239.

${ }^{23}$ S. F. Zhang, Phys. Rev. Lett. 83, 640 (1999).

${ }^{24}$ M. Julliere, Phys. Lett. 54A, 225 (1975).

${ }^{25} \mathrm{Ch}$. Binek and B. Doudin, J. Phys.: Condens. Matter 17, L39 (2005).

${ }^{26}$ H. Kohlstedt, N. A. Pertsev, J. Rodriguez Contreras, and R. Waser, Phys. Rev. B 72, 125341 (2005).

${ }^{27}$ Tianquan Lu and Wenwu Cao, Phys. Rev. B 66, 024102 (2002). 